\title{
Tumor Control and Overall Survival after Stereotactic Body Radiotherapy for Pulmonary Oligometastases from Colorectal Cancer: A Meta-Analysis
}

\author{
Hoon Sik Choi, MD, MS 1 \\ Bae Kwon Jeong, MD, PhD² \\ Ki Mun Kang, MD, PhD' \\ Hojin Jeong, $\mathrm{PhD}{ }^{2}$ \\ Jin Ho Song, MD, PhD ${ }^{3}$ \\ In Bong Ha, MD, MS² \\ Oh-Young Kwon, MD, PhD ${ }^{4}$
}

${ }^{1}$ Department of Radiation Oncology and Institute of Health Science, Gyeongsang National University Changwon Hospital, Gyeongsang National University College of Medicine, Changwon, ${ }^{2}$ Department of Radiation Oncology and Institute of Health Science, Gyeongsang National University Hospital, Gyeongsang National University College of Medicine, Jinju, 'B Department of Radiation Oncology, Seoul St. Mary's Hospital, College of Medicine, The Catholic University of Korea, Seoul, ${ }^{4}$ Department of Neurology and Institute of Health Science, Gyeongsang National University Hospital, Gyeongsang National University College of Medicine, Jinju, Korea

Correspondence: Oh-Young Kwon, MD, PhD Department of Neurology and Institute of Health Science, Gyeongsang National University Hospital, Gyeongsang National University College of Medicine, 79 Gangnam-ro, Jinju 52727, Korea

Tel: 82-55-750-8288

Fax: 82-55-750-1709

E-mail: oykwon@gnu.ac.kr

Received April 29, 2020

Accepted July 19, 2020

Published Online July 21, 2020

\begin{abstract}
Purpose
In pulmonary oligometastases from colorectal cancer (POM-CRC), the primarily recommended local therapy is metastasectomy. Stereotactic body radiotherapy (SBRT) is another local therapy modality that is considered as an alternative option in patients who cannot undergo surgery. The purpose of this meta-analysis is to demonstrate the effects of SBRT on POM-CRC by integrating the relevant studies.
\end{abstract}

\section{Materials and Methods}

The authors explored MEDLINE, EMBASE, Cochrane Library, Web of Science, and SCOPUS, and selected studies including patients treated with SBRT for POM-CRC and availability of local control (LC) or overall survival (OS) rate. In this meta-analysis, the effect of SBRT was presented in the form of the LC and OS rates for 1, 2, 3, and 5 years after SBRT as pooled estimates, and the frequency of pulmonary toxicity of grade 3 or higher after SBRT (PTG3SBRT).

\section{Results}

Fourteen full texts among the searched 4,984 studies were the objects of this meta-analysis. The overall number of POM-CRC patients was 495 as per the integration of 14 studies. The pooled estimate LC rate at 1, 2, 3, and 5 years after SBRT was $81.0 \%, 71.5 \%, 56.0 \%$, and $61.8 \%$, and the OS rate was $86.9 \%, 70.1 \%, 57.9 \%$, and $43.0 \%$, respectively. The LC and OS rates gradually declined until 3 years after SBRT in a similar pattern. Among the 14 studies, only two studies reported PTG3-SBRT as $2.2 \%$ and $10.8 \%$, respectively.

\section{Conclusion}

For POM-CRC, SBRT is an ablative therapy with a benefit on LC and OS rates and less adverse effects on the lung.

\section{Key words}

Colorectal neoplasms, Lung metastasis, Radiosurgery, Meta-analysis

\section{Introduction}

The limited number of metastatic tumors occurring at restricted sites are termed as oligometastases (OM). $\mathrm{OM}$ is an intermediate state of tumors, between widespread and localized, and implies the applicability of local therapy [1]. Unfor- tunately, colorectal cancer (CRC) recurs in more than half of patients even after definitive radical surgery [2,3]. In such patients, CRC recurs frequently in the form of OM in the liver and lung $[4,5]$. In patients with pulmonary OM from CRC (POM-CRC), metastasectomy along with systemic therapy leads to improvement in survival outcomes [6-10]. National 
Comprehensive Cancer Network (NCCN) and European Society for Medical Oncology (ESMO) groups also recommend metastasectomy as an ablative therapy for POM-CRC [11-13].

Patients with metastatic cancer often refuse surgery or are medically or surgically inoperable. Stereotactic body radiotherapy (SBRT) can be an alternative ablative option for metastasectomy because it allows precise irradiation to lesions and minimal radiation exposure to surrounding normal tissues. The NCCN and ESMO groups also recommend SBRT as an alternative treatment modality for $\mathrm{OM}$ patients who are incapable of undergoing surgery. To present the effects of SBRT for POM-CRC, several studies with various publication types including original study, systematic review, and metaanalysis have been published $[14,15]$. Although previous studies have shown excellent tumor control ability of SBRT, physicians still do not have a clear reason to choose SBRT over surgery for POM-CRC patients. To directly or indirectly compare the treatment effect of surgery and SBRT, it is important to investigate the overall survival (OS) after SBRT in detail based on every year and to grasp the association between local control (LC) and OS rate.

This meta-analysis aimed to investigate the LC and OS rates of POM-CRC patients after SBRT serially at yearly time points and to investigate the relationship between them. The other goal was to investigate the frequency of pulmonary toxicity of grade 3 or higher after SBRT (PTG3-SBRT).

\section{Materials and Methods}

\section{Search strategy}

A meta-analysis was performed in compliance with 'the Preferred Reporting Items for Systematic Review and MetaAnalysis (PRISMA)' guidelines [16]. The protocol of this meta-analysis has been submitted to The International prospective register of systematic reviews (PROSPERO) (CRD42020164111) [17].

To establish a strategy to find all studies that could be the subject of this meta-analysis as a study that applied SBRT as a local therapy to patients with POM-CRC and observed its outcome, appropriate search terms were selected. The words and phrases chosen were lung, pulmonary, metastasis, recurrence, colorectal, colon, sigmoid, rectal, cancer, carcinoma, tumor, neoplasm, radiosurgery, stereotactic body radiotherapy, stereotactic radiotherapy, stereotactic ablative radiotherapy, stereotactic radiosurgery, SBRT, stereotactic radiotherapy, and stereotactic ablative radiotherapy. While performing for relevant studies, the publication date chosen was from database inception to September 3, 2019. MEDLINE, EMBASE, Cochrane Library, Web of Science, and SCOPUS were the online databases used. Other bibliographies, such as reference lists and gray literature were also examined. There was no limitation with regard to publication date or language. EndNote X9.2 was the software used to handle the searched studies [18].

\section{Study selection}

For this meta-analysis, the following inclusion criteria were set: original researches, studies having information about CRC as primary cancer and pulmonary metastasis as $\mathrm{OM}$, and studies providing information on at least one of LC and $O S$ rates. The studies that included patients with concurrent uncontrolled extrathoracic metastasis when performing SBRT were excluded because it can significantly affect survival. If there were multiple reports from an institution and it seemed that the patient samplings included the same patients, only one study was left by applying the following priority principle: single topic, more sampling of patients, and more up-to-date study.

The searched data was sent to EndNote X9.2. Immediately after the sending, the automatic check function of EndNote removed duplicated studies. After removing the duplicated studies, the initial screening process of the searched data was embarked. In the initial screening process, the studies with irrelevant subjects were removed using titles and abstracts. The studies filtered in the initial screening process entered into the second selection process. In this course, the researchers reviewed the full texts of the screened studies carefully and selected studies that suited the purpose of this meta-analysis. In this process, four reviewers (H.S. Choi, B.K. Jeong, I.B. Ha, and K.M. Kang) worked independently. For the disagreements that occurred between the four reviewers, the authors reappraised the studies through discussion or consultation with other authors (H. Jeong and J.H. Song).

\section{Data synthesis and analysis}

Information extracted from the selected studies was general data (authors, publication years, study design, and OM definition), patient details (sample number, age, sex, tumor size, and follow-up duration), treatment profiles (SBRT dose, fractionation, and presence of concurrent chemotherapy) and outcome (LC rate, OS rate, and PTG3-SBRT).

For this meta-analysis, we used R ver. 3.5.3 (The R Foundation for Statistical Computing, Vienna, Austria) [19]. LC and OS rates were extracted as effect sizes to calculate pooled estimates at the time points of 1 year, 2 years, 3 years, and 5 years after SBRT for patients with POM-CRC. The selected studies were conducted on similar patients treated with standardized modalities and provided LC rate, OS rate, or both. Thus, we used a fixed-effects model for the meta-analysis as a predetermined way on the viewpoint that heterogeneity did not seem clinically significant among the studies. The studies included in this meta-analysis adopted one of the Common Terminology Criteria for Adverse Events (CTCAE) and Radiation Therapy Oncology Group (RTOG) scale to evaluate pulmo- 


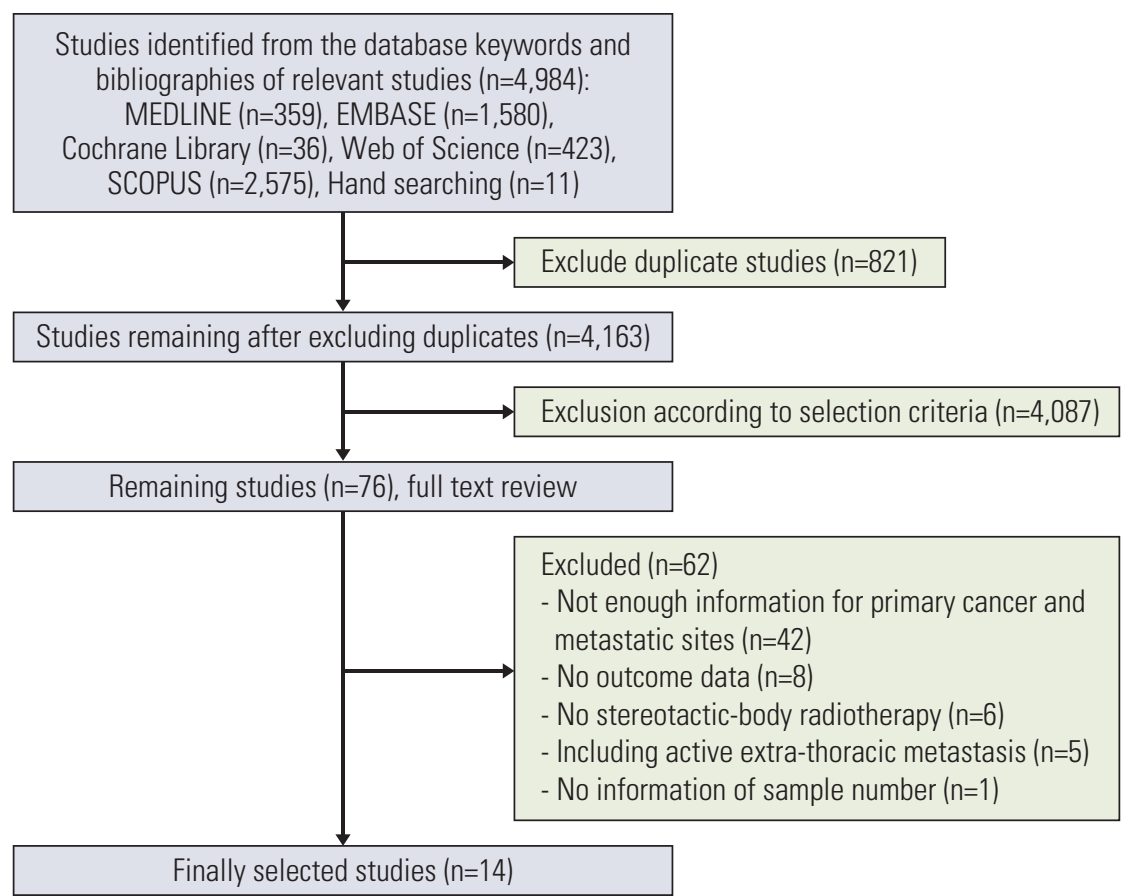

Fig. 1. Flow map for identification of relevant studies.

nary toxicity. The frequency of PTG3-SBRT was reviewed.

A forest plot for each meta-analysis was generated to display the findings. For visual evaluation of heterogeneity, the forest plots were used. Cochrane's $Q$ and $I^{2}$ were calculated for each analysis as statistical methods for measuring the heterogeneity. When the $p$-value of Cochran's $Q$ was less than 0.1 or the $\mathrm{I}^{2}$ was more than $50 \%$, heterogeneity was considered significant among the studies.

Eventually, 14 studies were selected in this meta-analysis. The authors, however, should perform different meta-analyses with recombination of the studies. The recombination was according to the types of outcomes and the time points of observation. As the number of studies incorporated in each meta-analysis was less than 10, evaluation of the publication bias was inappropriate in this review.

\section{Quality assessment of literature}

The Newcastle-Ottawa-Scale (NOS) is a checklist for assessing the quality of literature in meta-analyses for non-randomized studies [20]. In this meta-analysis, the cohort version NOS was used for quality assessment of the literature. When evaluating each of the finally selected studies as the NOS, two authors (H.S. Choi, O.Y. Kwon) independently evaluated and formed a consensus. The NOS for cohort studies consists of nine items grouped into three dimensions: selection, comparability, and outcome. It uses a star system that assesses each item and gives one star if the study has the highest quality for that. The only item of comparability can have two stars as an exception. Therefore, the number of stars that each study can have in the evaluation by the NOS ranges between $0-9$. The NOS scores 7-9 refer to high-quality and 4-6 refer to medium quality.

\section{Results}

\section{Identification of relevant studies}

Using the initially set search strategy, we collected 4,984 studies that could be the probable subject of research: 359 from MEDLINE, 1,580 from EMBASE, 36 from Cochrane Library, 423 from Web of Science, 2,575 from SCOPUS, and 11 from hand searching. Immediately after the collection, we excluded 821 duplicate studies. Through the initial screening process with the remaining 4,163 studies, 4,087 studies were eliminated from the data. Through the secondary selection process with the full texts of the remaining 76 studies, 62 studies were excluded for the following reasons: not enough information for being aware of primary cancer and metastatic sites ( $n=42)$, no outcome data $(n=8)$, no SBRT $(n=6)$, including active extrathoracic metastasis $(n=5)$, and no information about the sample number $(\mathrm{n}=1)$. Finally, 14 studies encompassing 495 patients with POM-CRC who underwent SBRT entered in this meta-analysis [21-34]. The process for study inclusion is presented in Fig. 1.

\section{Features of the included studies}

As shown in Table 1, 14 studies were selected for this metaanalysis. The publication year of the studies was between 

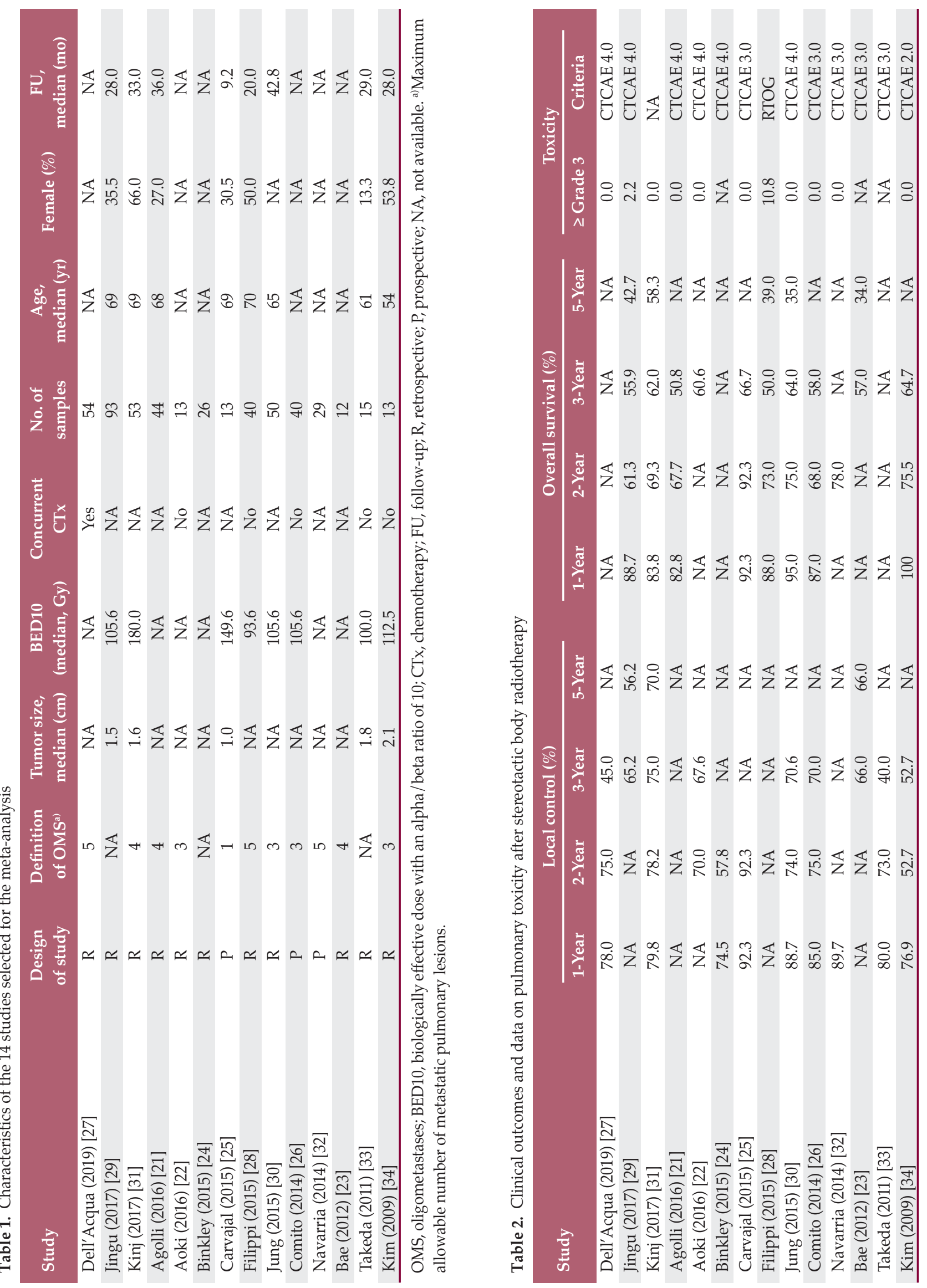

우

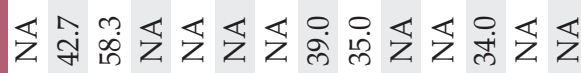

อิ

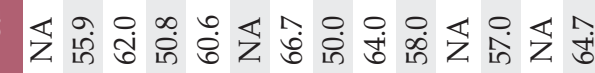

量

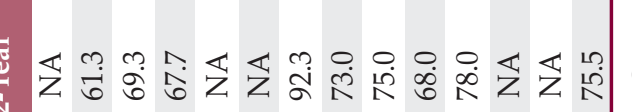

宛

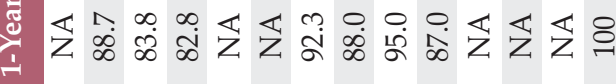

0
0
0
0
0

ذ)

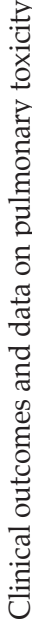

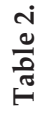

के

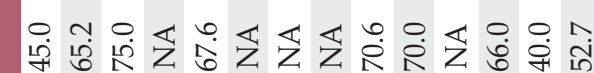

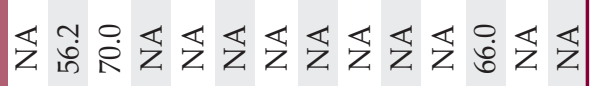

产

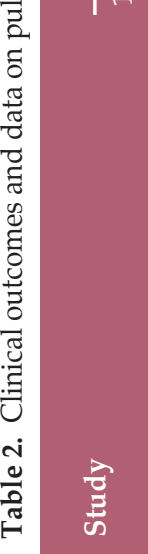

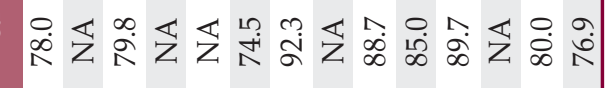

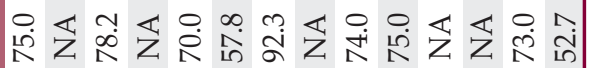

हี

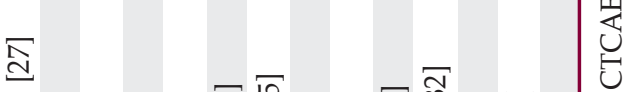

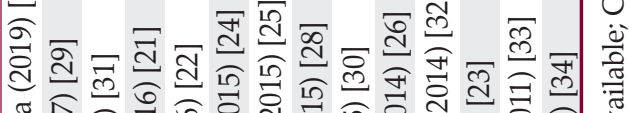

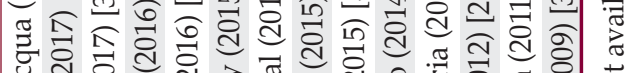

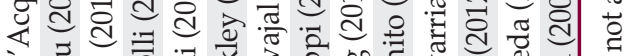

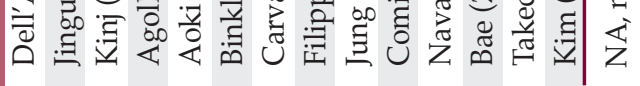




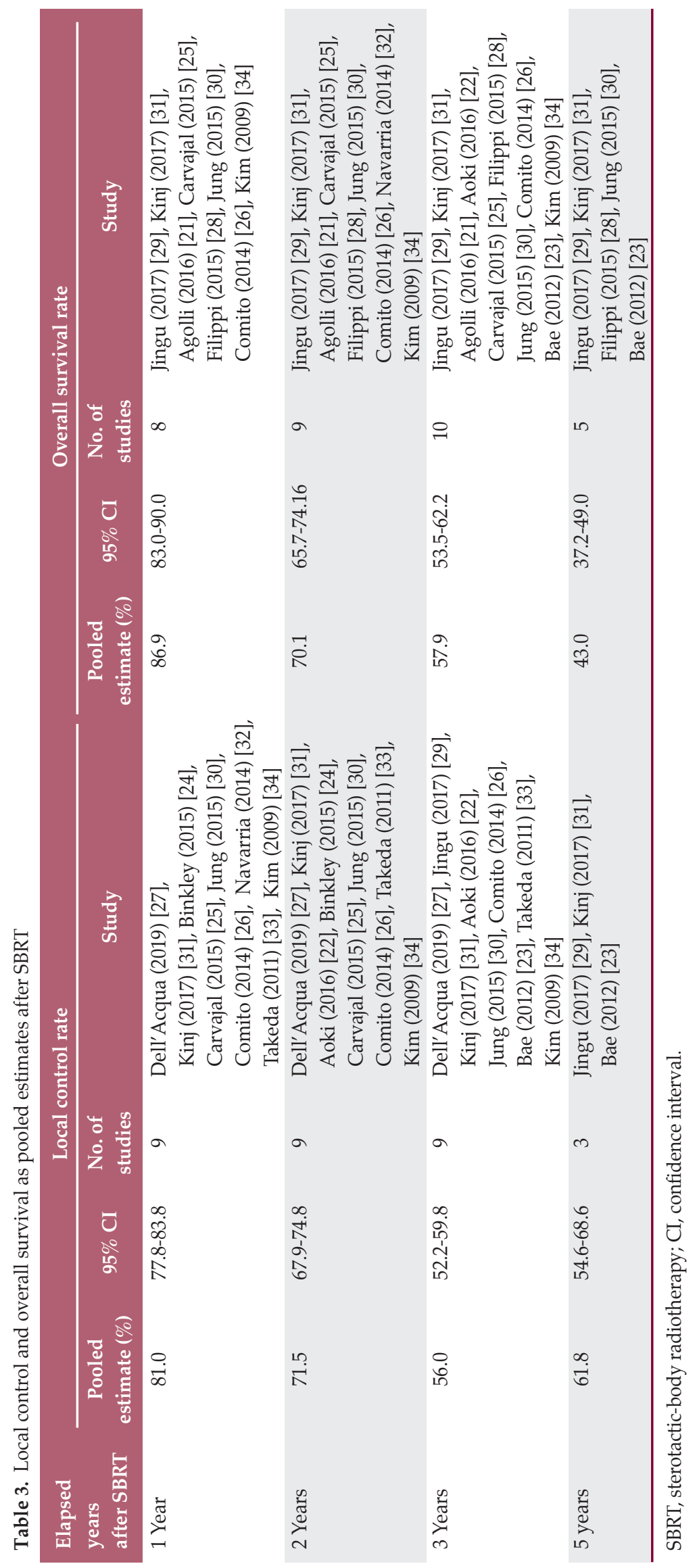




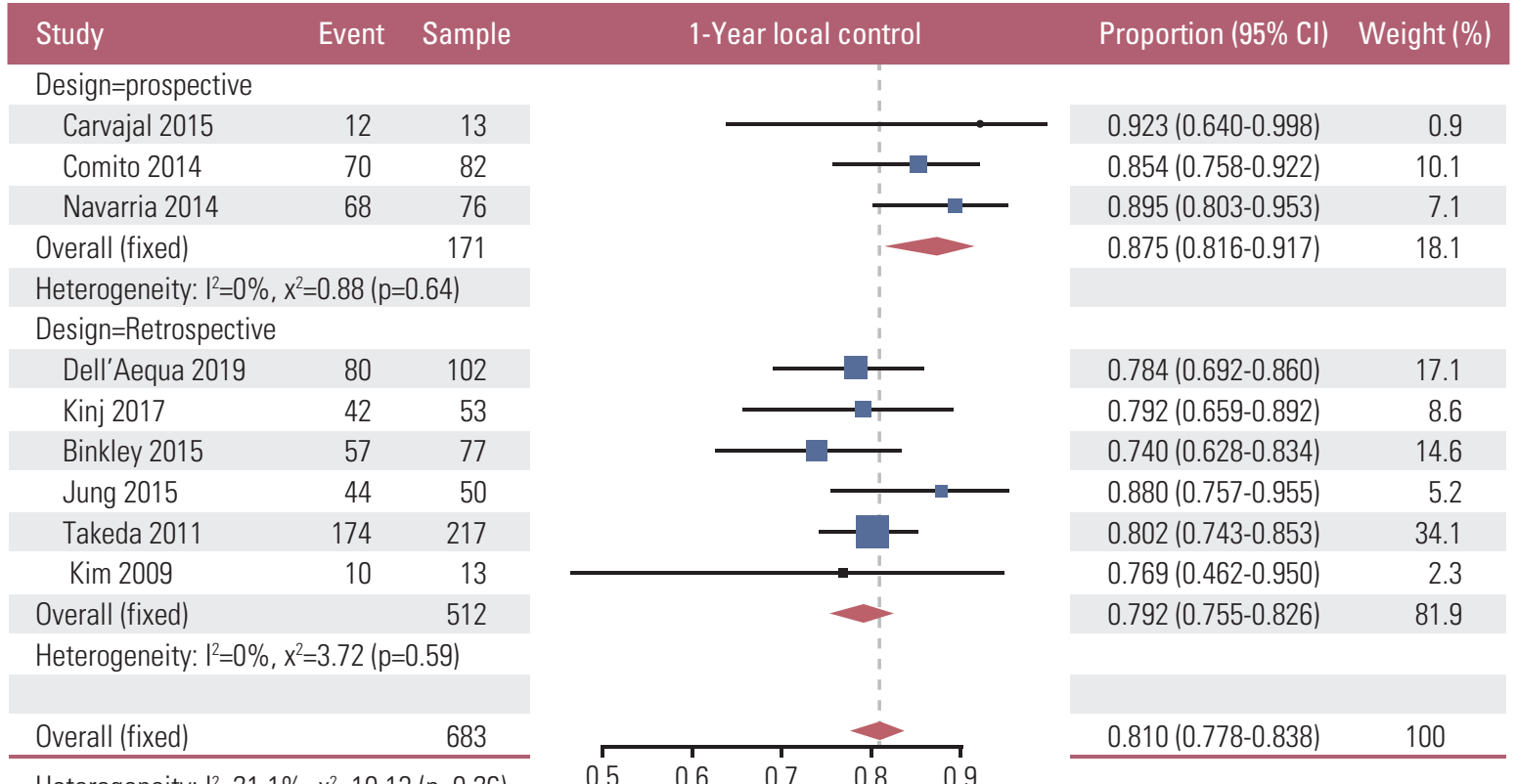

Heterogeneity: $I^{2}=21.1 \%, x^{2}=10.13(p=0.26)$

Residual heterogeneity: $I^{2}=0 \%, x^{2}=4.59(p=0.71)$

Fig. 2. Pooled estimates of local control rate at 1 year after stereotactic body radiotherapy [24-27,30-34]. CI, confidence interval.

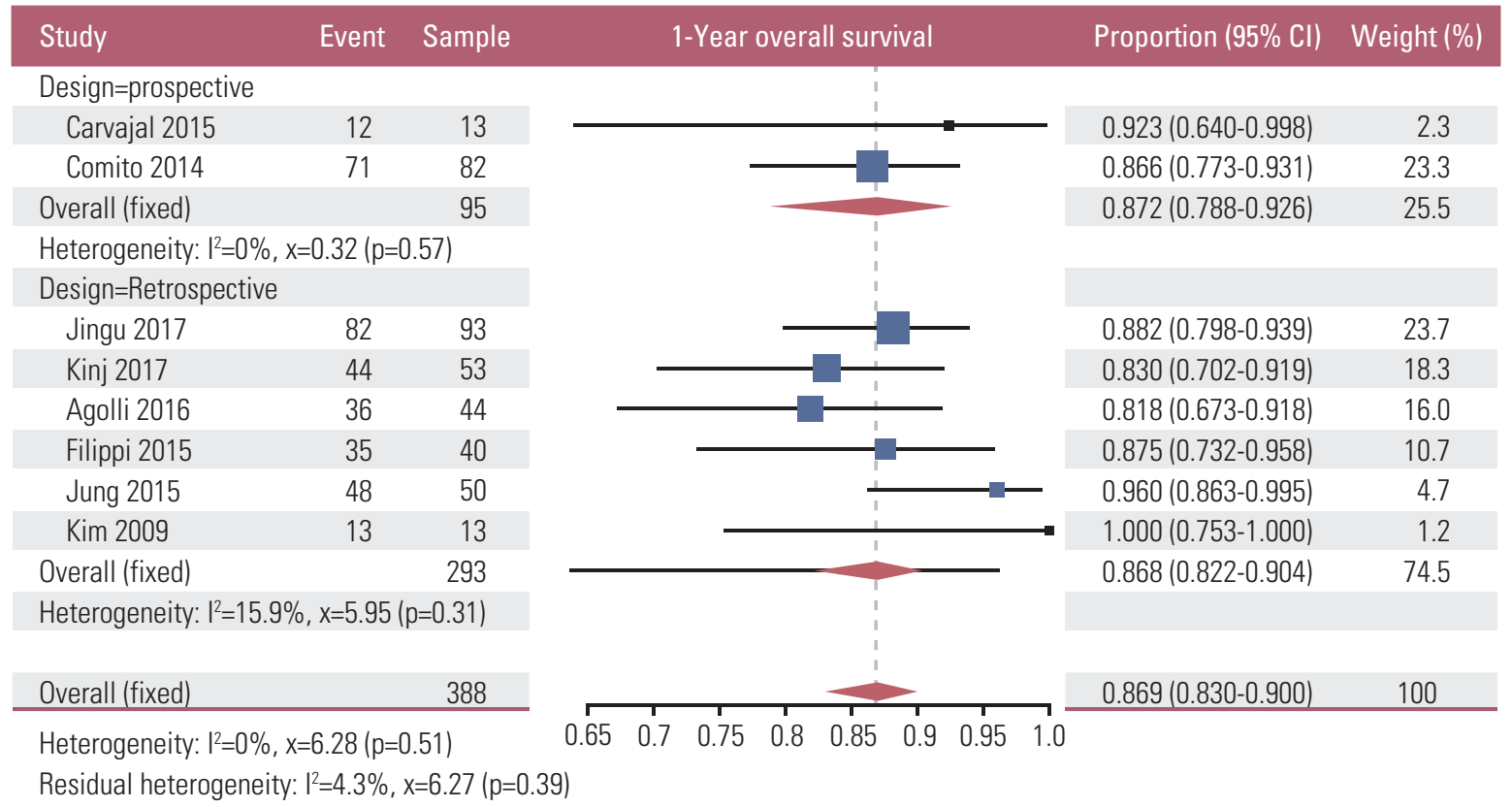

Fig. 3. Pooled estimates of overall survival rate at 1 year after stereotactic body radiotherapy $[21,25,26,28-31,34]$. CI, confidence interval.

2009 and 2019. The design was retrospective in 11 studies and prospective in the other three studies. Six of 14 studies reported whether chemotherapy was concurrently given, during the SBRT period: a study [27] allowed concurrent chemotherapy in the study design; and the other five studies $[22,26,28,33,34]$ excluded these patients from the analysis. OM was defined based on the number of lesions in 11 of
14 studies. The maximum allowable number of pulmonary lesions as $\mathrm{OM}$ in each study ranged from 1 to 5 ; the median was four. The median value of metastatic tumor size from five studies ranged from 1.0 to $2.1 \mathrm{~cm}$; the median was 1.6 $\mathrm{cm}$. The median prescription doses of SBRT collected from eight studies were converted based on a biologically effective dose with an alpha/beta ratio of 10 (BED10). The median of 
BED10 was 105.6 Gy and ranged from 93.6 to 180 Gy. For the finally selected 14 studies, the sample number varied from 12 to 93 and the median was 34.5. The median age of patients from eight studies ranged from 54 to 70 years, and the median of the medians was 68.5 years. The median percentage of females was $35.5 \%(13.3 \%-66.0 \%)$ among the seven studies, which provided sex distribution. The median followup duration in eight studies ranged from 9.2 to 42.8 months, and the median was 28.5 months.

The effect sizes of LC and OS rate at 1, 2, 3, and 5 years after SBRT were extracted from each primary study and shown in Table 2. The median rate of $\mathrm{LC}$, at 1, 2, 3, and 5 years was $80.0 \%(74.5 \%-92.3 \%, \mathrm{n}=9), 74.0 \%(52.7 \%-92.3 \%, \mathrm{n}=9), 66.0 \%$ $(40.0 \%-75.0 \%, \mathrm{n}=9)$, and $66.0 \%(56.2 \%-70.0 \%, \mathrm{n}=3)$, respectively. The median rate of OS at 1, 2, 3, and 5 years was $88.4 \%(82.8 \%-100.0 \%, \mathrm{n}=8), 73.0 \%(61.3 \%-92.3 \%, \mathrm{n}=9), 59.3 \%$ $(50.0 \%-66.7 \%, \mathrm{n}=10)$, and $39.0 \%(34.0 \%-58.3 \%, \mathrm{n}=5)$, respectively. All the finally selected studies provided information on the frequency of PTG3-SBRT. As criteria for evaluating the toxicity of SBRT, the CTCAE was applied in 12 studies (ver. 2.0 for one, ver. 3.0 for five, and ver. 4.0 for six) and the RTOG in one study. The remaining study did not provide information about the criteria. There were two of 14 studies that provided information about PTG3-SBRT. In a study using the CTCAE as an evaluation tool, PTG3-SBRT occurred in 2.2\% of patients [29]. Another study, which evaluated the toxicity based on RTOG, reported PTG3-SBRT as a dense radiographic appearance in $10.8 \%$ of patients, but all the patients were asymptomatic [28].

\section{Pooled estimates}

The pooled estimates of the LC and the OS rate at 1, 2, 3 , and 5 years after SBRT are shown in Table 3. Nine studies reported the LC rate at 1 year after SBRT, with a pooled estimate of $81.0 \%$ (95\% confidence interval [CI], 77.8 to 83.8 ); for this, $\mathrm{I}^{2}$ was $21.1 \%$, and Q-value was $10.13(\mathrm{p}=0.26)$. The estimate was $87.5 \%$ (95\% CI, 81.6 to 91.7 ) in three prospective studies and $79.2 \%$ (95\% CI, 75.5 to 82.6$)$ in six retrospective studies in the subgroup analysis performed according to the study designs. The Q-value was 5.54 between the subgroups $(\mathrm{p}=0.019)$ and 4.59 within the subgroups $(\mathrm{p}=0.71)$ (Fig. 2). Combination of nine studies revealed the pooled estimate of the LC rate at 2 years after SBRT as 71.5\% (95\% CI, 67.9 to 74.8$), \mathrm{I}^{2}$ was $37.9 \%$, and Q-value was $12.88(\mathrm{p}=0.12)$. The pooled estimate was $77.0 \%$ (95\% CI, 67.3 to 84.5 ) for two prospective studies and 70.7\% (95\% CI, 66.8 to 74.3 ) for seven retrospective studies in the subgroup analysis. $\mathrm{Q}$ was 1.53 $(p=0.22)$ between the groups and $11.35(p=0.12)$ within the groups. The pooled estimate at 3 years after SBRT was $56.0 \%$ (95\% CI, 52.2 to 59.8) when collecting LC rates at 3 years after SBRT from 9 studies. $I^{2}$ was $84.8 \%$ and Q-value was 52.72 $(\mathrm{p}<0.01)$ for this analysis. One of the nine studies was a prospective study and eight were retrospective. While collecting

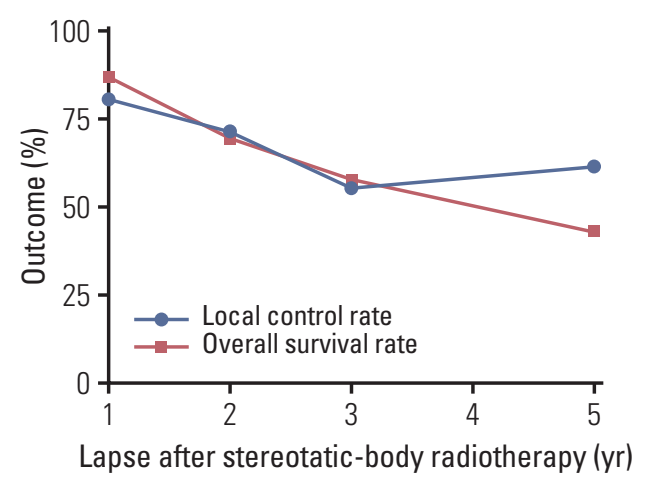

Fig. 4. Local control and overall survival at 1, 2, 3, and 5 years after stereotactic body radiotherapy with trend lines.

three retrospective studies reporting LC at 5 years after SBRT, the pooled estimate, $\mathrm{I}^{2}$, and Q-value were $61.8 \%(95 \% \mathrm{CI}, 54.6$ to 68.6$), 34.8 \%$, and 3.07 ( $\mathrm{p}=0.22)$, respectively.

Eight out of 14 studies reported OS rate at 1 year after SBRT, and the pooled estimate, $\mathrm{I}^{2}$, and Q-value were $86.9 \%$ (95\% CI, 83.0 to 90.0$), 0.0 \%$, and $6.28(\mathrm{p}=0.51)$, respectively (Fig. 3). In the subgroup analysis for the study design, the pooled estimate was $87.2 \%$ (95\% CI, 78.8 to 92.6 ) for two prospective studies out of the eight studies and $86.8 \%$ (95\% CI, 82.2 to 90.4 ) for the other six retrospective ones. $\mathrm{Q}$ was 0.01 ( $\mathrm{p}=0.92)$, between the subgroups and $6.27(\mathrm{p}=0.39)$ within the subgroups (Fig. 3). Nine studies reported OS rate at 2 years after SBRT. The pooled estimate was 70.1\% (95\% CI, 65.7 to 74.2$), \mathrm{I}^{2}$ was $14.1 \%$, and $\mathrm{Q}$-value was $9.32(\mathrm{p}=0.32)$. The pooled estimate was $73.4 \%$ (95\% CI, 66.1 to 79.6 ) for the three prospective studies out of the nine studies and 68.3\% (95\% $\mathrm{CI}, 62.7$ to 73.4$)$ for the other six retrospective ones. Q-value was $1.27(\mathrm{p}=0.26)$ between the subgroups and $8.04(\mathrm{p}=0.33)$ within the subgroups. Ten studies provided afforded OS rate at 3 years after SBRT. The pooled estimate was $57.9 \%$ (95\% CI, 53.5 to 62.2 ), $\mathrm{I}^{2}$ was $0.0 \%$, and Q-value was 4.46 ( $\mathrm{p}=0.88$ ). For the subgroup analysis, the pooled estimate was $59.9 \%$ (95\% CI, 49.8 to 69.3) for the two prospective studies out of the 10 studies and $57.4 \%$ (95\% CI, 52.5 to 62.2$)$ for the other eight retrospective studies. Q-value was $0.19(\mathrm{p}=0.66)$ between the subgroups and was $4.27(\mathrm{p}=0.81)$ within the subgroups. The pooled OS rate was $43.0 \%$ (95\% CI, 37.2 to 49.0 ) at 5 years after SBRT, and $\mathrm{I}^{2}$ was 46.4 , and Q-value was $7.46(\mathrm{p}=0.11)$ as per the five retrospective studies.

A line graph showing the serial rates of LC and OS was created to observe the relation between them (Fig. 4). The trend line of LC showed a gradual decrease pattern until three years after the SBRT and no further decrease at five years after SBRT. Meanwhile, that of OS showed a continuous decrease until five years after SBRT. Both the trend lines showed very similar changes up to 3 years after SBRT. 


\section{Study quality assessment}

A star for the item 'selection of the non-exposed cohort' and 'comparability' for all studies while assessing the quality of primary studies could not be provided in this metaanalysis through NOS due to the absence of controls in all the studies. In six studies, the follow-up period was less than 2 years and it was judged that the periods were not sufficient for observing the outcome and toxicity. A star could not be given for six studies for the item 'length of outcome' in NOS. As a result, six out of 14 studies subjected to this metaanalysis received five stars in the NOS evaluation, and the remaining eight studies received six stars. Therefore, all subjects of this meta-analysis had medium quality. The results of the assessment are provided in S1 Table.

\section{Discussion}

A meta-analysis was conducted by collecting 14 studies that reported the effect of SBRT for POM-CRC. Overall, 495 patients were included in the study. The average number of metastatic lung lesions was 1.4 per patient, and the median prescription dose of SBRT for the POM-CRC was 105.6 Gy of BED10. The primary goal of this meta-analysis was to combine LC and OS rates over time after SBRT for POM-CRC. The investigated time points were 1 year, 2 years, 3 years, and 5 years after SBRT. For each time points, the LC rate was $81.0 \%, 71.5 \%, 56.0 \%$, and $61.8 \%$, respectively, and the OS rate was $86.9 \%, 70.1 \%, 57.9 \%$, and $43.0 \%$, respectively. A gradual decrease in the LC and OS rates was observed with a similar pattern from 1 year to 3 years after SBRT. However, after 3 years, only the OS rate decreased and no change was observed in the LC rate. Only two studies reported PTG3-SBRT with a frequency of $2.2 \%$ and $10.8 \%$, respectively.

NCCN and ESMO groups prefer metastasectomy as an ablative therapy for POM-CRC and consider SBRT as another option when surgery is not possible. Several retrospective studies have reported the effect of pulmonary metastasectomy in POM-CRC patients with a 5-year OS rate of $32.4 \%$ $43 \%$ [7-10]. A meta-analysis involving 25 retrospective studies reported the effects of lung metastasectomy in POM-CRC patients with OS rate as a range value and the 5-year OS rate as 27\%-68\% [6]. A randomized clinical trial demonstrated the effect of metastasectomy for POM-CRC, the researchers compared the outcome of patients between a group with metastasectomy plus chemotherapy, and chemotherapy only as controls. At five years after treatment, the estimated survival rate was $38 \%$ and $29 \%$ for the metastasectomy group and controls, respectively [35]. As the study was terminated prematurely with only 65 participants due to recruiting difficulty, the data has weak evidence. Nonetheless, the information helps to assess the meaning of the present meta-analysis. The present meta-analysis revealed that the 5-year survival rate was $43 \%$ among the POM-CRC patients treated with SBRT. Although most of the primary studies included in this meta-analysis included the patients whose conditions were too poor to endure the operation in their cohort, the effects of SBRT for POM-CRC seemed comparable to metastasectomy effects demonstrated by previous studies. The information provided by the present meta-analysis suggests a new perspective that the SBRT can be a valuable option as a primary treatment of POM-CRC.

Despite comparable treatment outcomes, there are several obstacles for selecting SBRT as the first choice of ablative therapy for POM-CRC. Compared to other primary cancers, the presence of numerous hypoxic cells may make POM-CRC more radioresistant [36]. Clinical studies on SBRT in POMCRC or POM from other primary cancers reported that the former had a lower LC rate after SBRT than the latter [33,37]. Nevertheless, it has become increasingly evident that higher radiation doses can surpass CRC radioresistance. A few studies have shown a significant benefit of the SBRT dose more than 100 Gy or 120 Gy (BED10) for the LC rate of POM-CRC $[24,25,29]$. Another study also found a dose-dependent effect of SBRT with BED10 more than 120 Gy for the OM that occurred in the liver, lymph nodes, and lung [23].

It is not clear if the LC derived by SBRT improves the survival rate in the POM-CRC patients [38]. Theoretically, SBRT, a local therapy method similar to surgery, can improve survival. The information provided by this meta-analysis provides a cue to get a solution to this issue. From yearly outcome information to the third year after SBRT, the sequential changes in the LC and OS rates are similar. Accordingly, up to 3 years after SBRT, the LC derived from SBRT in POM$\mathrm{CRC}$ patients is hypothesized to have an association with the OS. Conversely, after 3 years, survival may be inhibited by other factors such as extrapulmonary metastases or the patient's general condition than the POM control, so additional survival improvement could be obtained through appropriate systemic therapy with supportive care.

Lung lesions move concordantly with the breath of the patients. Therefore, when physicians use radiotherapy as a treatment modality of POM-CRC, there is a concern that it is difficult to irradiate the correct location on the lesion. However, advances in the SBRT technique have put forward more effective and less toxic treatment by delivering high radiation dose to the targets and stiff dose gradient between normal tissues. Our meta-analysis also showed that SBRT is a less toxic and safe treatment option.

A previously reported systematic review gathered studies that investigated the effect of SBRT on POM-CRC without providing pooled estimates through meta-analysis [14]. Two previously reported meta-analyses investigated the effects of SBRT on POM-CRC. One of them presented pooled estimates as the odds ratio of local failure [39]. The other metaanalysis reported the LC, OS, and progression-free survival 
rates after SBRT for POM-CRC [15]. Compared to information provided by the previous studies, our meta-analysis has an additional strength. For consistent and comprehensive integration, the studies whose cohort included the patients having active metastatic lesions outside the lung were excluded. The pooled estimates of the LC and OS rates obtained through the integration of the present meta-analysis may make the outcome information of POM-CRC treated with SBRT clearer.

The compositions were identical among the 14 studies chosen for the present meta-analysis. The primary studies enrolled in the present meta-analysis seemed not to vary in their characteristics. First, from the time we selected the studies, the inclusion criteria were set to recruit studies with possible homogeneity. All the primary studies had data for the POM-CRC patients without metastatic lesions when treated with SBRT. In particular, the authors selected only the studies that used the standardized SBRT with breath correction without concurrent chemotherapy and only the studies that reported LC and OS as outcomes. After collecting the studies, it was also possible to discover that there was homogeneity somewhat, among the studies. The average age of the patients included in each study was similar. Eight studies were available to obtain information on average age, with a distribution of age 54-70. In six of these studies, the mean age of the patient group was 65 or older. The design of the study also showed a low degree of heterogeneity among the included studies. Of the 14 subjects, 11 had a retrospective design, and only three had a prospective design. The quality assessment of the literature evaluated by the NOS also revealed low heterogeneity. The NOS scores of all the 14 research studies were 5-6 points, which was the medium quality. So, the authors presumed that the clinical heterogeneity was low and chose the fixed-effects model as a preset mode for the statistical method. The choice seemed appropriate, as the heterogeneity was also weak in terms of statistical figures and forest plots. Just at three years after SBRT, the LC rate showed substantial heterogeneity with an $\mathrm{I}^{2}$ value of $88 \%$. Nevertheless, it seemed to be a statistical coincidence because the heterogeneities of other meta-analyses, including the same studies, were low.

The present review has a limitation that most of the studies included in the meta-analysis were retrospective. Another limitation is that all the included studies lacked proper controls. Since studies comparing the SBRT treatment group with the surgical treatment group or chemotherapy only group were not in the finally selected studies, it was difficult to demonstrate whether SBRT improved outcomes statistically. Such limitations may have originated in a clinical environment that is difficult for researchers to overcome. It would be challenging to envision randomized controlled studies, given the cancer patient's right to choose the treatment. Despite such difficulties, more reliable information could be obtained if additional prospective studies emerge added later, and scholars gather and meta-analyze them.

In conclusion, we demonstrated that SBRT has been useful for the treatment of POM-CRC throughout this meta-analysis. This review demonstrates the relationship between LC and OS rates and the probable presence of a similar trend of gradual decrement up to 3 years after SBRT. Besides, this review shows that SBRT-related pulmonary toxicity might be acceptable for the treatment of POM-CRC. It is suggested that SBRT in POM-CRC could be an effective and safe ablative therapy and option for metastasectomy.

\section{Electronic Supplementary Material}

Supplementary materials are available at Cancer Research and Treatment website (https://www.e-crt.org).

\section{Conflicts of Interest}

Conflict of interest relevant to this article was not reported.

\section{References}

1. Hellman S, Weichselbaum RR. Oligometastases. J Clin Oncol. 1995;13:8-10.

2. Elias D, Benizri E, Vernerey D, Eldweny H, Dipietrantonio D, Pocard M. Preoperative criteria of incomplete resectability of peritoneal carcinomatosis from non-appendiceal colorectal carcinoma. Gastroenterol Clin Biol. 2005;29:1010-3.

3. Galandiuk S, Wieand HS, Moertel CG, Cha SS, Fitzgibbons RJ $\mathrm{Jr}$, Pemberton JH, et al. Patterns of recurrence after curative resection of carcinoma of the colon and rectum. Surg Gynecol Obstet. 1992;174:27-32.

4. Lee WS, Yun SH, Chun HK, Lee WY, Yun HR, Kim J, et al. Pulmonary resection for metastases from colorectal cancer: prognostic factors and survival. Int J Colorectal Dis. 2007;22:699704.
5. Van Cutsem E, Nordlinger B, Adam R, Kohne CH, Pozzo C, Poston $\mathrm{G}$, et al. Towards a pan-European consensus on the treatment of patients with colorectal liver metastases. Eur J Cancer. 2006;42:2212-21.

6. Gonzalez M, Poncet A, Combescure C, Robert J, Ris HB, Gervaz P. Risk factors for survival after lung metastasectomy in colorectal cancer patients: a systematic review and meta-analysis. Ann Surg Oncol. 2013;20:572-9.

7. Pfannschmidt J, Muley T, Hoffmann H, Dienemann H. Prognostic factors and survival after complete resection of pulmonary metastases from colorectal carcinoma: experiences in 167 patients. J Thorac Cardiovasc Surg. 2003;126:732-9.

8. Saito Y, Omiya H, Kohno K, Kobayashi T, Itoi K, Teramachi M, et al. Pulmonary metastasectomy for 165 patients with colo- 
rectal carcinoma: a prognostic assessment. J Thorac Cardiovasc Surg. 2002;124:1007-13.

9. Yedibela S, Klein P, Feuchter K, Hoffmann M, Meyer T, Papadopoulos $\mathrm{T}$, et al. Surgical management of pulmonary metastases from colorectal cancer in 153 patients. Ann Surg Oncol. 2006;13:1538-44.

10. Zampino MG, Maisonneuve P, Ravenda PS, Magni E, Casiraghi M, Solli $\mathrm{P}$, et al. Lung metastases from colorectal cancer: analysis of prognostic factors in a single institution study. Ann Thorac Surg. 2014;98:1238-45.

11. National Comprehensive Cancer Network. Rectal cancer (version 1. 2020) [Internet]. Plymouth Meeting, PA: National Comprehensive Cancer Network; 2020 [cited 2020 Jul 19]. Available from: https://www.nccn.org/professionals/physician_gls/ pdf/rectal.pdf.

12. National Comprehensive Cancer Network. Colon cancer (version 1. 2020) [Internet]. Plymouth Meeting, PA: National Comprehensive Cancer Network; 2020 [cited 2020 Jul 19]. Available from: https://www.nccn.org/professionals/physician_gls/ pdf/colon.pdf.

13. Van Cutsem E, Cervantes A, Adam R, Sobrero A, Van Krieken $\mathrm{JH}$, Aderka D, et al. ESMO consensus guidelines for the management of patients with metastatic colorectal cancer. Ann Oncol. 2016;27:1386-422.

14. Kobiela J, Spychalski P, Marvaso G, Ciardo D, Dell'Acqua V, Kraja F, et al. Ablative stereotactic radiotherapy for oligometastatic colorectal cancer: systematic review. Crit Rev Oncol Hematol. 2018;129:91-101.

15. Cao C, Wang D, Tian DH, Wilson-Smith A, Huang J, Rimner A. A systematic review and meta-analysis of stereotactic body radiation therapy for colorectal pulmonary metastases. J Thorac Dis. 2019;11:5187-98.

16. Moher D, Liberati A, Tetzlaff J, Altman DG; PRISMA Group. Preferred reporting items for systematic reviews and metaanalyses: the PRISMA statement. Ann Intern Med. 2009;151: 264-9.

17. Booth A, Clarke M, Dooley G, Ghersi D, Moher D, Petticrew $\mathrm{M}$, et al. The nuts and bolts of PROSPERO: an international prospective register of systematic reviews. Syst Rev. 2012;1:2.

18. Clarivate Analytics. Endnote X9.2. Philadelphia, PA: Clarivate Analytics; 2019.

19. R Core Team. R: A language and environment for statistical computing [Internet]. Vienna: R Foundation for Statistical Computing; 2019 [cited 2020 Jul 19]. Available from: https:// www.R-project.org/.

20. Wells G, Shea B, O'Connell D, Peterson J, Welch V, Losos M, et al. The Newcastle-Ottawa Scale (NOS) for assessing the quality of norandomized wtudies in meta-analysis [Internet]. Ottawa: Ottawa Hospital Research Institue; 2000 [cited 2020 Jul 19]. Available from: http://www.ohri.ca/programs/clinical_epidemiology/oxford.asp.

21. Agolli L, Bracci S, Nicosia L, Valeriani M, De Sanctis V, Osti MF. Lung metastases treated with stereotactic ablative radiation therapy in oligometastatic colorectal cancer patients: outcomes and prognostic factors after long-term follow-up. Clin Colorectal Cancer. 2017;16:58-64.

22. Aoki M, Hatayama Y, Kawaguchi H, Hirose K, Sato M, Akimoto $\mathrm{H}$, et al. Stereotactic body radiotherapy for lung metas- tases as oligo-recurrence: a single institutional study. J Radiat Res. 2016;57:55-61.

23. Bae SH, Kim MS, Cho CK, Kang JK, Kang HJ, Kim YH, et al. High dose stereotactic body radiotherapy using three fractions for colorectal oligometastases. J Surg Oncol. 2012;106: 138-43.

24. Binkley MS, Trakul N, Jacobs LR, von Eyben R, Le QT, Maxim $P G$, et al. Colorectal histology is associated with an increased risk of local failure in lung metastases treated with stereotactic ablative radiation therapy. Int J Radiat Oncol Biol Phys. 2015;92:1044-52.

25. Carvajal C, Navarro-Martin A, Cacicedo J, Ramos R, Guedea F. Stereotactic body radiotherapy for colorectal lung oligometastases: preliminary single-institution results. J BUON. 2015;20:158-65.

26. Comito T, Cozzi L, Clerici E, Campisi MC, Liardo RL, Navarria $\mathrm{P}$, et al. Stereotactic ablative radiotherapy (SABR) in inoperable oligometastatic disease from colorectal cancer: a safe and effective approach. BMC Cancer. 2014;14:619.

27. Dell'Acqua V, Surgo A, Kraja F, Kobiela J, Zerella MA, Spychalski $\mathrm{P}$, et al. Stereotactic radiation therapy in oligometastatic colorectal cancer: outcome of 102 patients and 150 lesions. Clin Exp Metastasis. 2019;36:331-42.

28. Filippi AR, Badellino S, Ceccarelli M, Guarneri A, Franco P, Monagheddu C, et al. Stereotactic ablative radiation therapy as first local therapy for lung oligometastases from colorectal cancer: a single-institution cohort study. Int J Radiat Oncol Biol Phys. 2015;91:524-9.

29. Jingu K, Matsuo Y, Onishi H, Yamamoto T, Aoki M, Murakami $Y$, et al. Dose escalation improves outcome in stereotactic body radiotherapy for pulmonary oligometastases from colorectal cancer. Anticancer Res. 2017;37:2709-13.

30. Jung J, Song SY, Kim JH, Yu CS, Kim JC, Kim TW, et al. Clinical efficacy of stereotactic ablative radiotherapy for lung metastases arising from colorectal cancer. Radiat Oncol. 2015;10:238.

31. Kinj R, Bondiau PY, Francois E, Gerard JP, Naghavi AO, Leysalle A, et al. Radiosensitivity of colon and rectal lung oligometastasis treated with stereotactic ablative radiotherapy. Clin Colorectal Cancer. 2017;16:e211-20.

32. Navarria P, Ascolese AM, Tomatis S, Cozzi L, De Rose F, Mancosu P, et al. Stereotactic body radiotherapy (sbrt) in lung oligometastatic patients: role of local treatments. Radiat Oncol. 2014;9:91.

33. Takeda A, Kunieda E, Ohashi T, Aoki Y, Koike N, Takeda T. Stereotactic body radiotherapy (SBRT) for oligometastatic lung tumors from colorectal cancer and other primary cancers in comparison with primary lung cancer. Radiother Oncol. 2011;101:255-9.

34. Kim MS, Yoo SY, Cho CK, Yoo HJ, Choi CW, Seo YS, et al. Stereotactic body radiation therapy using three fractions for isolated lung recurrence from colorectal cancer. Oncology. 2009;76:212-9.

35. Treasure T, Farewell V, Macbeth F, Monson K, Williams NR, Brew-Graves C, et al. Pulmonary Metastasectomy versus Continued Active Monitoring in Colorectal Cancer (PulMiCC): a multicentre randomised clinical trial. Trials. 2019;20:718.

36. van Laarhoven HW, Kaanders JH, Lok J, Peeters WJ, Rijken $\mathrm{PF}$, Wiering $\mathrm{B}$, et al. Hypoxia in relation to vasculature and 
proliferation in liver metastases in patients with colorectal cancer. Int J Radiat Oncol Biol Phys. 2006;64:473-82.

37. Franceschini D, Cozzi L, De Rose F, Navarria P, Franzese C, Comito T, et al. Role of stereotactic body radiation therapy for lung metastases from radio-resistant primary tumours. J Cancer Res Clin Oncol. 2017;143:1293-9.

38. Siva S, MacManus M, Ball D. Stereotactic radiotherapy for pulmonary oligometastases: a systematic review. J Thorac Oncol. 2010;5:1091-9.

39. Jingu K, Matsushita H, Yamamoto T, Umezawa R, Ishikawa Y, Takahashi N, et al. Stereotactic radiotherapy for pulmonary oligometastases from colorectal cancer: a systematic review and meta-analysis. Technol Cancer Res Treat. 2018;17: 1533033818794936. 